\title{
Indirect RSVP for Virtual Cluster Cellular Mobile IP Networks
}

\author{
Yu Zeng, Jon W. Mark, and Xuemin Shen \\ University of Waterloo, Waterloo, Ontario, Canada N2L 3G1 \\ \{yzeng, jwmark, xshen\}@bbcr.uwaterloo.ca
}

\begin{abstract}
Mobile IP (MIP) could be deployed in a wireless cellular network so that the integrated Cellular Mobile IP (CMIP) network can be used to support datagram delivery to mobile users. In order to provide QoS for realtime traffic in IP network, Resource reSerVation Protocol (RSVP) is needed to reserve network resources along the route which the datagrams follow. However, current RSVP fails to operate through the MIP tunnel. Moreover, the high registration rate in a CMIP network greatly degrades RSVP performance. In this paper, an Indirect RSVP (IRSVP) over Virtual Cluster Cellular Mobile IP (VCCMIP) is proposed in which an assistant RSVP connection is introduced to assist the endto-end major RSVP operation over the MIP tunnel. IRSVP signaling costs and packet loss rates are evaluated. Simulation results show that the proposed scheme can greatly increase RSVP performance in terms of packet loss and RSVP connection active percentage.
\end{abstract}

\section{Introduction}

The importance of Internet to present day society hardly needs reiteration. As the backbone network to support user roaming, it does create many challenging problems. In a hybrid wireless/IP-based network, connection establishment and provision for continuous communications between mobile hosts across the internet require unique identification of Internet Protocol (IP) addresses before any actual communication can begin. However, in an environment where the mobile hosts constantly change their access points, an interworking infrastructure and a networking protocol are needed to support mobility without disrupting any ongoing communication. Unfortunately, the current suite of internet protocols (TCP/IP) fall short of mobility support since they are designed under the assumption that the end hosts are fixed. A Mobile IP (MIP) [1]-2] protocol has been specified by the Internet Engineering Task Force (IETF) to support host mobility in an IP network. It enables a mobile host to have two IP addresses, one for identification and the other for routing. MIP can be implemented over current cellular networks, namely Cellular Mobile IP (CMIP), to extend IP services to mobile users. In CMIP, base stations (BSs) serve as MIP home or foreign agents to assist packet forwarding. However, high mobility tends to trigger frequent IP address change and MIP registration update. 
IP network provides "best effort" services. It does not guarantee Quality of Service (QoS) of multimedia traffic. In order to meet the pre-designed QoS required by realtime traffic, Resource reSerVation Protocol (RSVP) [3]-4] is proposed to reserve network bandwidth along the route which IP packets follow. However, RSVP fails to reserve end-to-end resources from the correspondent host to the mobile host in a CMIP network because of the presence of MIP tunnel. Either a new scheme or a modification to RSVP is needed to operate over the CMIP network.

In this paper, an Indirect RSVP (IRSVP) scheme for a Virtual Cluster Cellular Mobile IP (VCCMIP) network is proposed. This scheme enables RSVP operation over a CMIP network, and achieves low registration rate. The remainder of the paper is organized as follows. Section 2 gives a brief overview of MIP, CMIP and RSVP. The IRSVP and VCCMIP concepts are presented in Section 3 . Simulation results and discussions on the performance of the proposed scheme are given in Section 4, and concluding remarks are given in Section 5.

\section{Overview of MIP, CMIP and RSVP}

\section{$2.1 \quad$ Mobile IP}

Several entities and addresses are defined in MIP. They are

- Mobile Host $(\mathrm{MH})$ - A host that can migrate from one network to another without changing its IP address, while maintaining any ongoing communications.

- Home Agent (HA) - A router with an interface on the MH's home network; the MH must update its home agent of its current location as it roams.

- Foreign Agent (FA) — A router with an interface on the MH's visited network which assists the home agent to deliver datagrams to the $\mathrm{MH}$ while it is away from its home.

- Correspondent Host $(\mathrm{CH})$ - A host that an $\mathrm{MH}$ is communicating.

- Home Address - An IP address assigned to an MH for permanent identification. It remains unchanged regardless of where the $\mathrm{MH}$ is located.

- Care-of Address - An IP address that is temporarily assigned to an MH when it is away from its home network. The $\mathrm{MH}$ may change its care-of address when it roams across the internet.

Mobile IP operates in the following way: home and foreign agents make themselves known by sending agent advertisement messages. Upon receiving an agent advertisement, the $\mathrm{MH}$ determines whether it is on its home network or a foreign network. An MH basically sends and receives packets like any other fixed host on its home network when it is at home. When the $\mathrm{MH}$ is away from its home network, it obtains a care-of address on the foreign network for the agent advertisement. The $\mathrm{MH}$ registers each new care-of address with its HA, possibly via its FA.

There are two types of routing in MIP as shown in Fig. 1, 
- Triangle Routing - Packets sent by a $\mathrm{CH}$ to an $\mathrm{MH}$ connected to a foreign link are routed first to the MH's HA and then tunneled to the MH's current care-of address using IP encapsulation. On the other hand, packets sent by the mobile node are routed directly to the correspondent.

- Optimized Routing — The HA informs the CH of the MH's care-of address and have the packets tunneled directly to the MH, bypassing the HA.

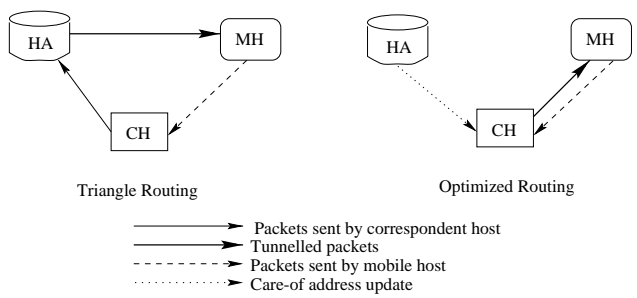

Fig. 1. Triangle routing vs. optimized routing

\subsection{Cellular Mobile IP}

CMIP implements MIP over a cellular network in the way that each BS acts as a $\mathrm{HA}$ or FA to assist packet forwarding. It is assumed in the CMIP network that:

- each and every MH, whether it is physically served by the wireline backbone IP network or the wireless cellular network, is identified by an unique IP address. The same rule applies to the $\mathrm{CH}$.

- The CMIP network supports packet data communication between BSs. BSs are also identified by IP addresses.

- HA and FA have the same functionalities as defined in MIP. MIP registration occurs whenever an $\mathrm{MH}$ moves out of a BS service region.

\section{$2.3 \quad$ RSVP}

RSVP is a simplex, receiver-oriented resource reservation setup protocol designed for an Integrated Services Internet (IntServ). RSVP is used by a host to request specific QoS from the network for particular application data streams or flows. RSVP requests generally result in resources being reserved in each node along the data path. RSVP reserves resources for RSVP sessions. An RSVP session is defined as

$$
<\text { session }>::=<\text { DstAddr, PID, DstPort }>\text {, }
$$

where $D s t A d d r$ is the destination IP address of the data packets, PID is the IP protocol ID, and DstPort is the destination UDP/TCP port. RSVP signaling messages are sent hop-by-hop between RSVP-capable routers as raw IP datagrams with protocol ID 46, which is reserved for RSVP signaling. An RSVP message consists of a common header, followed by a variable number of "object". 
- Common header: The main purpose of the common header is to define the type of RSVP message. Seven types of RSVP messages have been defined so far, the most important two are Path and Resv messages, which will be discussed later.

- Object: Objects carry necessary information needed to perform RSVP signaling. For example, a $<$ session $>$ object defines a particular session. $R S V P_{-} H O P$ object carries the IP address of the RSVP-capable node that sends this message.

A typical RSVP message looks like

$$
<\text { RSVPMsg }>::=<\text { Header }><\text { Object } 1><\text { Object } 2>\cdots .
$$

As shown in Fig. 2, a successful RSVP setup involves at least the exchange of two RSVP messages between a sender and a receiver, a Path message from the sender to the receiver to provide path information to all RSVP-capable routers, and a Resv message from the receiver to the sender to reserve network resources. Path and Resv messages are in the form of

$$
<\text { PathMsg/ResvMsg }>::=<\text { Header }><\text { Session }><\text { RSVP_HOP }>\cdots .
$$

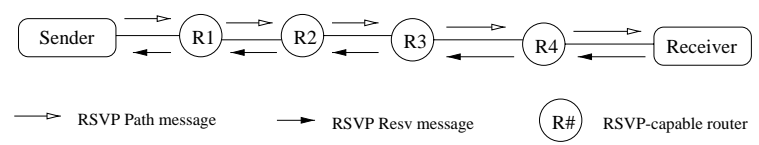

Fig. 2. Resource reservation process

Suppose the sender in Fig. 2 wants to reserve resource to the receiver for its data flow, it sends a Path message

$$
<\text { PathMsg }>::=<\text { Header }><\text { RAddr, PID, DstPort }><\text { SAddr }>\cdots,
$$

where $R A d d r$ and $S A d d r$ are IP addresses of the receiver and the sender, respectively. When RSVP-capable router R1 receives this Path message, it

- caches path information carried by the Path message, and

- sends out another Path message

$$
<\text { PathMsg }>::=<\text { Header }><R A d d r, \text { PID, DstPort }><R 1 \text { Addr }>\cdots
$$

to the next router.

The Path message is only used to carry RSVP path information to all routers along the data path. No resources are reserved at this stage. Once the receiver gets the Path message, it starts the reservation by replying with a Resv message

$$
<\text { ResvMsg }>::=<\text { Header }><\text { RAddr, PID, DstPort }><\text { RAddr }>\cdots
$$


to the sender. According to the pre-cached path information in those routers, this Resv message follows exactly the reverse path of the Path message. When $\mathrm{R} 4$ receives the Resv message, it reserves resources in itself accordingly and sends another Resv message to the next hop. After the Resv message is processed by all routers and the sender, resource reservation completes.

\section{Indirect RSVP}

Unlike the current IP network in which a host must have a fixed network interface in order to keep its ongoing IP connections alive, CMIP enables an MH to roam within the network while maintaining its IP layer connection so that any ongoing communication will not be interrupted. However, as illustrated in Section 3.1. RSVP over CMIP fails because of tunneling inherent in MIP. This deficiency can be rectified by isolating the tunnel and implementing RSVP over the tunnel separate from the rest of the return path. We refer to this mode of RSVP as Indirect RSVP (IRSVP). The operation of IRSVP is described in Section 3.3.

\subsection{RSVP over CMIP}

Since RSVP is a simplex protocol and CMIP has two routing schemes, several cases need to be studied separately as shown in Table 1

Table 1. RSVP over CMIP operation cases

\begin{tabular}{ccc}
\hline Packet direction & $\mathrm{MH} \rightarrow \mathrm{CH} \mathrm{MH} \leftarrow \mathrm{CH}$ \\
\hline Triangle routing & Case 1 & Case 2 \\
\hline Optimized routing & Case 3 & Case 4 \\
\hline
\end{tabular}

- Case 1: As shown in Fig. 31(a), to reserve resources from the MH to the CH, the MH sends RSVP Path message to the $\mathrm{CH}$ in the form of

$<$ PathMsg $>::=<$ Header $><$ CHAddr, PID, DstPort $><$ MH HomeAddr $>$,

in which $<C H A d d r$, PID, DstPort $>$ is the desired RSVP session. As mentioned before, this Path message is sent out as a raw IP packet with protocol ID 46. The intermediate RSVP-capable routers process the Path message accordingly. Upon receiving the Path message, the $\mathrm{CH}$ starts resource reservation by sending an RSVP Resv message

$$
<\text { ResvMsg }>::=<\text { Header }><C H A d d r, \text { PID, DstPort }><C H A d d r>\cdots .
$$

Then a proper route as shown in Fig. 3(b) is reserved when the MH receives the Resv message. Data flows follow exactly the reserved route. Therefore, RSVP works well in Case 1. 


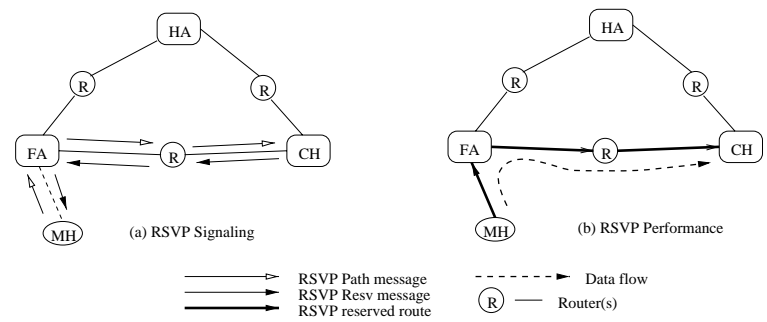

Fig. 3. RSVP over MIP: Case 1

- Case 2: Figure 4(a) shows RSVP signaling in Case 2 in which the $\mathrm{CH}$ is the sender. The Path message sent by the $\mathrm{CH}$,

$<$ PathMsg $>::=<$ Header $><$ MHHomeAddr, PID, DstPort $><$ CHAddr $>$,

can arrive at the HA properly. However, according to MIP, when the HA intercepts any packet destined to an $\mathrm{MH}$, it encapsulates the packet in another IP packet with protocol ID 4 (reserved for IP encapsulation) and forwards it to the MH. The intermediate routers between the HA and FA identify RSVP Path message by checking whether the protocol ID is 46. As a result, these encapsulated Path messages are invisible to those routers so that no RSVP path informations are cached. In any case, this Path message can still arrive at the MH. The FA will be able to cache RSVP path information after decapsulation, but the previous RSVP_HOP is the HA. The MH responds to the Path message with an RSVP Resv message. Upon the FA's receiving of the Resv message, an RSVP route is reserved from the FA to the MH. Since the FA will send Resv message to the HA according to the path information cached, no route is reserved from the HA to the FA, but the route from the $\mathrm{CH}$ to the HA is still reserved. The tunnel seems like a non-RSVP cloud. RSVP fails to operate over the MIP tunnel in this case as shown in Fig. 4(b).
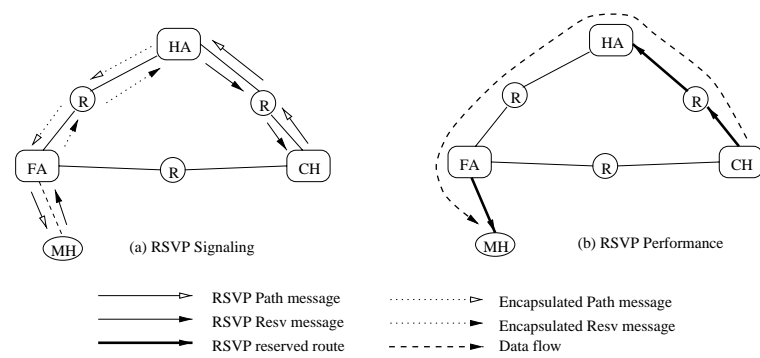

Fig. 4. RSVP over CMIP: Case 2 
- Case 3 - Case 3 operates the same way as Case 1 since packets are routed the same way from $\mathrm{MH}$ to the $\mathrm{CH}$ no matter whether triangle or optimized routing scheme is used.

- Case 4 - Similar RSVP signaling for Case 2 happens to Case 4. RSVP fails to reserve resources from the $\mathrm{CH}$ to the $\mathrm{FA}$ as shown in Fig. 5 .
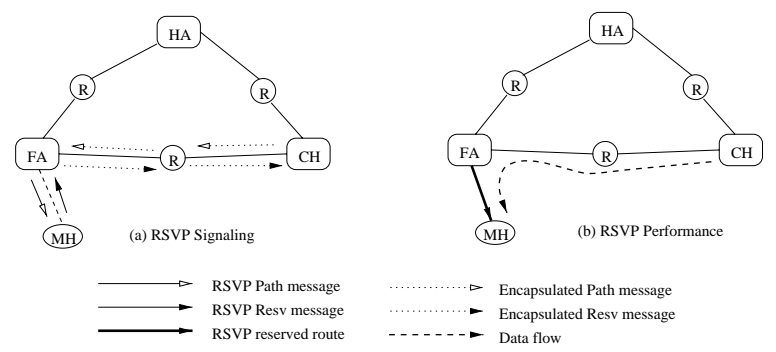

Fig. 5. RSVP over CMIP: Case 4

In summary, RSVP works well in a CMIP environment to reserve network resources from the $\mathrm{MH}$ to the $\mathrm{CH}$, but it fails in the opposite direction since the presence of the MIP tunnel makes RSVP Path message invisible to routers along the tunnel.

\subsection{Mobility Impact}

Mobility is another factor that degrades packet delivery performance in a CMIP network. When an $\mathrm{MH}$ moves to another BS/FA, it triggers the following events:

- hard handoff: The hard handoff involves the physical and data link layer connections to be established. Any upper layer communication cannot start until the connections are established.

- registration and address update: Once the $\mathrm{MH}$ detects that it has moved, it obtains a new care-of address, and starts an MIP registration by sending out registration request and waiting for registration reply. If optimized routing is used, care-of address in all possible CHs also need to be updated. Any IP layer communication cannot start until the above processes complete.

- RSVP route update: Although RSVP supports automatic adaptive route change, it takes time to update the new route. The worst case happens when there is address changes. Since RSVP session is identified by the triple: DstAddr, PID and PortNum, any address change will cause packets not to be recognized by RSVP-capable routers any more. Therefore, any address change results in the entire RSVP route being torn down and re-reserved, which will greatly degrade QoS of realtime traffic.

On the other hand, any new scheme can benefit from 
1. reducing the handoff or registration rate, or both,

2. accelerating the registration and address update processes, and

3. introducing fast RSVP route update scheme.

The proposed IRSVP scheme improves packets delivery performance by taking advantage of item 1 and 3 . The rest of this subsection discusses the proposed registration rate reduction scheme, while the next subsection describes the operation of the IRSVP scheme.

Although it is straight-forward in a CMIP network to construct FAs at BSs where wireless and wireline links conjunct, it endures high MIP registration rate because whenever handoff occurs, registration takes place, that registration rate equals handoff rate. In the proposed Virtual Cluster Cellular Mobile IP (VCCMIP), MSCs act as FAs. All MHs associated with the same MSC are configured as one subnetwork, which includes several BSs. Handoff happens when the MH moves out of the service area covered by its current serving MSC. Therefore, registration happens at a much lower rate than handoff. The actual registration rate depends on the number of BSs controlled by an MSC, and the MH's mobility pattern.

\subsection{Operation of Indirect RSVP}

In IRSVP, the end-to-end RSVP connection is composed of two RSVP segments, a major RSVP connection excluding the MIP tunnel and an assistant RSVP connection through the tunnel. This section will focus on Case 2 only because a similar approach can be implemented for Case 4 .

As shown in Fig. 6(a), when an RSVP-capable HA receives a Path message from a $\mathrm{CH}$, it

1. first encapsulates the Path message and forwards it to the $\mathrm{MH}$ to perform the major RSVP reservation,

2. then sends out a new RSVP Path message to the FA to start an assistant RSVP setup. This Path message is sent without encapsulation in the form of

$$
<\text { PathMsg }>::=<\text { Header }><F A A d d r, \text { PID, DstPort }><H A A d d r>\cdots .
$$

PID and the DstPort are copied from the major RSVP Path message, while it changes the session by replacing the $M H A d d r$ with the FAAddr. The other fields of the Path message are set accordingly.

3. The HA also creates a session binding $<$ MHHomeAddr, PID, DstPort $>\longrightarrow<$ FAAddr, PID, DstPort $>$ so that the major RSVP session will be mapped to the assistant RSVP session.

When the FA receives the major and assistant Path messages, it

1. caches RSVP path information according to the major Path messages,

2. forwards the decapsulated major Path messages to the $\mathrm{MH}$, 

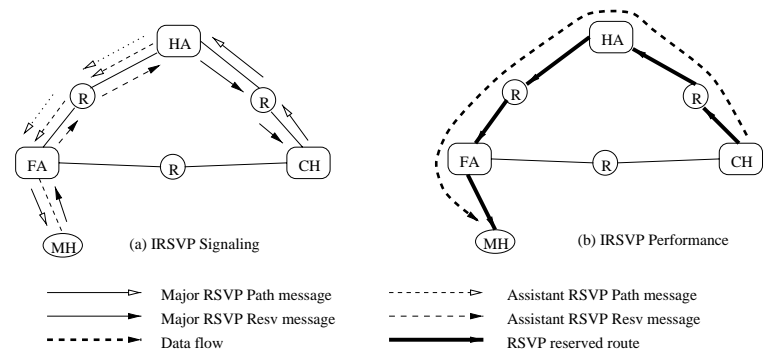

Fig. 6. Indirect RSVP

3. replies to the assistant Path messages with a Resv messages in the form of $<$ ResvMsg $>::=<$ Header $><$ FAAddr, PID, DstPort $><$ FAAddr $>\cdots$.

to reserve resources in the assistant RSVP segment, and

4. creates another session binding $<F A A d d r$, PID, DstPort $>\longrightarrow$

$<$ MHHomeAddr, PID, DstPort $>$ so that the assistant RSVP session will be mapped back to the major RSVP session.

When the HA intercepts any RSVP session related data packets, it does not use IP encapsulation to route those packets. The HA simply changes the original destination IP address, which is the MH's home address, to the FA's IP address. This makes it possible for the intermediate routers along the tunnel to recognize RSVP sessions. In this way, the end-to-end RSVP connection is achieved by combining two separate RSVP connections with proper session mapping, as shown in Fig. 6(b).

\section{Simulation and Discussion}

\subsection{Mobility Model}

For simulation simplicity, assume a wireless service area is tilted by square cells. Each virtual cluster is composed of $N$ radio cells. The $\mathrm{MH}$ mobility pattern is modeled as a Markov chain. Every $T$ seconds, the test MH either stays with the same BS with probability $P_{\text {same }}$, or hands off to adjacent BSs with probability Pother, and

$$
P_{\text {same }}+P_{\text {other }}=1,
$$

It is further assumed that if handoff happens, the $\mathrm{MH}$ is equally likely to hand off to any of the four adjacent cells. The MH registers its new care-of address with its HA only when it moves out of its current MSC service region. The network cost metrics used in the simulation for the performance evaluation are given in Table 2, Most of them are cited from [6] and [7]. 
Table 2. Network cost metrics

\begin{tabular}{lc}
\hline Description & Value \\
\hline Cell size & $800 \mathrm{~m}$ \\
\hline Average speed of MHs & $60 \mathrm{~km} / \mathrm{h}$ \\
\hline RSVP Path message process time & $3 \mathrm{~ms}$ \\
\hline RSVP Resv message process time & $5 \mathrm{~ms}$ \\
\hline Session mapping access time & $3 \mathrm{~ms}$ \\
\hline Tunneling and detunneling time & $7 \mathrm{~ms}$ \\
\hline Average Internet packet delay per hop & $20 \mathrm{~ms}$ \\
\hline Average wireless link delay & $40 \mathrm{~ms}$ \\
\hline To generate a control message & $5 \mathrm{~ms}$ \\
\hline Agent advertisement period & $50 \mathrm{~ms}$ \\
\hline RSVP Path and Resv message update period & $30 \mathrm{~s}$ \\
\hline
\end{tabular}

\subsection{Simulation Results}

1. Registration rate - Figure 7 shows the registration rates in the CMIP and the proposed VCCMIP schemes when one MSC controls 4, 9 and $16 \mathrm{BSs}$. It is observed that there is significant registration rate drop in VCCMIP, which benefits realtime traffic.

2. Overall RSVP signaling cost - Figure 8 shows the overall RSVP signaling cost for RSVP-CMIP and the proposed IRSVP-VCCMIP schemes. The signaling cost is measured by the processing time needed to maintain an RSVP connection, which indicates the signaling complexity of the scheme. The time to generate control message, to process RSVP Path and Resv messages, and to access session mappings are considered in the simulation. Although it can be seen that the IRSVP scheme endures higher signaling cost because of the extra signaling cost associated with the assistant RSVP connection, the cost is still relatively small.

3. Percentage RSVP active time - Figure 9 shows the RSVP connection active percentage, which is defined as the percentage of time that the RSVP connection is alive, i.e., capable of transmitting packets. In the simulation, there are seven hops from the $\mathrm{CH}$ to the $\mathrm{HA}$, from the HA to the FA, and two hops from the FA to the MH. It is worth to indicate that the IRSVP scheme greatly increases the RSVP active percentage, especially in a highly mobile environment (small $P_{\text {same }}$ ).

4. Average packet loss rate - Figure 10 shows the packet loss rate vs. the RSVP Path message update period. Although short update periods brings low loss rates, it will sharply increase RSVP signaling cost. It is observed that IRSVP achieves much lower loss rate than RSVP. By comparing the two figures, it is also seen that low registration rate is important to obtain good performance. 


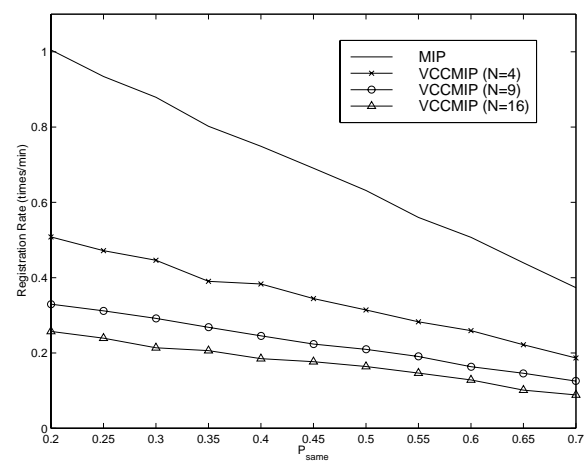

Fig. 7. Registration rate in CMIP and VCCMIP

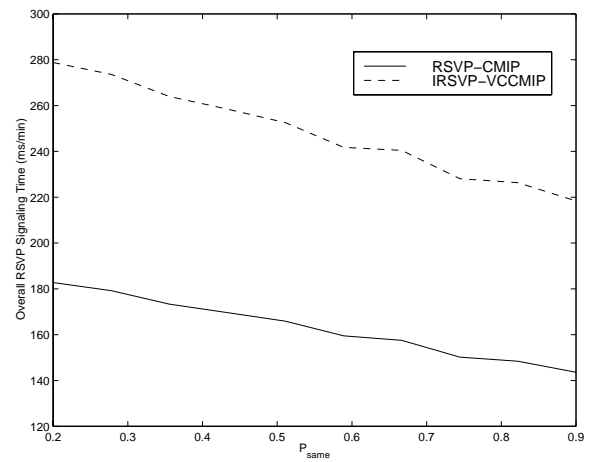

Fig. 8. Overall RSVP signaling cost

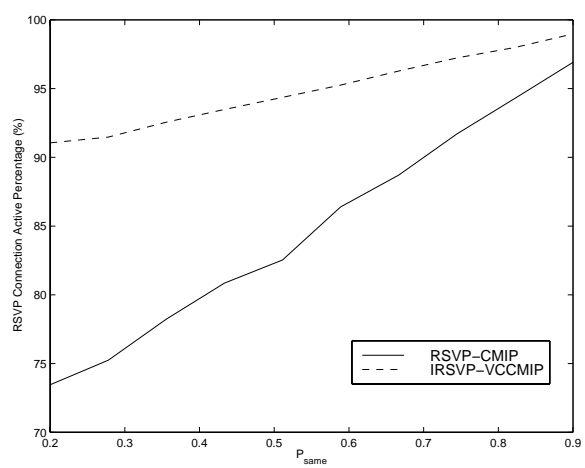

Fig. 9. RSVP connection active percentage 

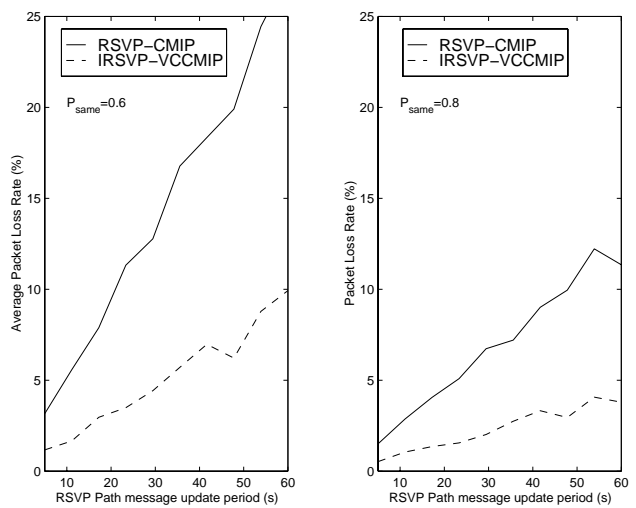

Fig. 10. Average packet loss rate

\section{Conclusion}

Since the RSVP-CMIP scheme fails to operate through the MIP tunnel and RSVP performance is greatly degraded in the presence of high registration rates, an IRSVP-VCCMIP scheme is proposed to address these problems. Analyses and simulation results show that the proposed scheme can increase RSVP performance in terms of packet loss and RSVP connection active percentage, while sustains an increase in signaling time. IRSVP performance may be further improved if a proper scheme is introduced in future work to accelerate the registration process, especially in the cellular network.

\section{Acknowledgments}

This work has been supported by the Natural Sciences and Engineering Research Council (NSERC) of Canada under grant No. RGPIN7779.

\section{References}

1. C. Perkins, "IP Mobility Support," RFC 2002, October 1996.

2. J. D. Solomon, Mobile IP. Prentice Hall, Inc., 1997.

3. R. Braden, L. Zhang, S. Berson, S. Herzog, and S. Jamin, "Resource ReSerVation Protocol (RSVP) -Version 1 Functional Specification," RFC 2205, September 1997.

4. P. P. White, "RSVP and Integrated Services in the Internet: A Tutorial," IEEE Communications Magazine, May 1997.

5. J. W. Mark, X. Shen, Y. Zeng, and J. Pan, "Issues in Wireless/IP Based Network," to appear in IEEE $3 G$ Wireless, June 2000.

6. K. W. Ng and V. C. M. Leung, "An IPv6-based Location Management Scheme for Client-Server Computing over Mobile Data Networks," IEEE WCNC, 1999.

7. T. J. Kostas, M. S. Borella, I. Sidhu, G. M. Schuster, J. Grabiec, and J. Mahler, "Real-Time Voice over Packet-Switched Networks," IEEE Network, January/February 1998. 